\title{
Prenatal Diagnosis of Isovaleric Acidemia from Amniotic Fluid Using Genetic and Biochemical Approaches
}

\section{Si Ding}

Shanghai Jiaotong University School of Medicine Xinhua Hospital

Lili Liang

Shanghai Jiaotong University School of Medicine Xinhua Hospital

Wenjuan Qiu

Shanghai Jiaotong University School of Medicine Xinhua Hospital

\section{Huiwen Zhang}

Shanghai Jiaotong University School of Medicine Xinhua Hospital

\section{Bing Xiao}

Shanghai Jiaotong University School of Medicine Xinhua Hospital

\section{Liping Dong}

Zibo Central Hospital

\section{Wenjun Ji}

Shanghai Jiaotong University School of Medicine Xinhua Hospital

\section{Feng Xu}

Shanghai Jiaotong University School of Medicine Xinhua Hospital

\section{Zhuwen Gong}

Shanghai Jiaotong University School of Medicine Xinhua Hospital

\section{Xuefan Gu}

Shanghai Jiaotong University School of Medicine Xinhua Hospital

\section{Lei Wang}

Shanghai Jiaotong University School of Medicine Xinhua Hospital

\section{Lianshu Han ( $\nabla$ hanlianshu@xinhuamed.com.cn )}

Xinhua Hospital, School of Medicine, Shanghai Jiao Tong University. https://orcid.org/0000-0003-4209-5119

\section{Research Article}

Keywords: Genetic analysis, Isovaleric Acidemia, Mass spectrometry, Metabolite analysis, Prenatal diagnosis

Posted Date: January 17th, 2022

DOI: https://doi.org/10.21203/rs.3.rs-1242418/v1

License: () (1) This work is licensed under a Creative Commons Attribution 4.0 International License. Read Full License 


\section{Abstract}

Background冈Isovaleric acidemia (IVA) is an inborn error of leucine metabolism and different approaches have been applied to its prenatal diagnosis. However, systemic application of a biochemical strategy is rare. To evaluate its reliability and validity, we conducted a retrospective study of our experience with metabolite measurement together with genetic analysis in IVA prenatal diagnosis at a single center.

Methods $₫ A$ total of eight pregnancies whose probands were diagnosed as IVA were referred to our center for prenatal diagnosis. Prenatal data of genetic analysis and metabolite measurement using tandem mass spectrometry (MS/MS) and gas chromatography/mass spectrometry (GC/MS) in amniotic fluid (AF) samples were retrospectively reviewed.

Results: Genetic and biochemical results were both available in these eight at-risk fetuses. Among them, two fetuses had higher levels of isovalerylcarnitine (C5) and C5/acetylcarnitine (C2) in AF compared with normal reference range and, thus, were determined to be affected, both of whom were found to carry compound heterogeneous mutations according to genetic analysis. The remaining six fetuses were determined to be unaffected based on a normal AF metabolite profile, except one showed slightly elevated C5 and they were found to be carriers according to genetic analysis. However, the level of isovalerylglycine (IVG) could not be detected at all in both groups. The diagnostic sensitivities of C5 and C5/C2 were both $100 \%$, and the specificities were $83.3 \%$ and $100 \%$, respectively.

Conclusions: The biochemical analysis, as a quick and convenient method, could be an additional reliable option for the prenatal diagnosis of IVA, especially in families with inconclusive genetic results, and can achieve a more precise diagnosis in conjunction with mutation analysis. Among the three tested biochemical markers, $\mathrm{C} 5 / \mathrm{C} 2$ appears to be the most credible.

\section{Introduction}

Isovaleric Acidemia (IVA, OMIM\#243500) is the first organic acidemia recognized in humans. The incidence of IVA ranges hugely from 1:33282 to $1: 622489$ in the worldwide ${ }^{[1-3]}$, and varies from regions to regions in China, which was reported to be 1:365,000 in Taiwan $^{[4]}, 1: 123,400$ in Zhejiang province ${ }^{[5]}$ and 1:84,469 in Quanzhou district ${ }^{[6]}$. IVA is an autosomal recessive inborn error of leucine metabolism caused by a deficiency of the isovaleryl-CoA dehydrogenase (IVD; E.C.1.3.99.10) enzyme ${ }^{[7]}$. Its deficiency results from mutations in the IVD gene, leading to the accumulation of derivatives of isovaleryl-CoA that are detected as isovalerylcarnitine (C5), isovaleric acid, 3-hydroxyisovaleric acid, and isovalerylglycine (IVG) in the cells, blood and urine ${ }^{[8,9]}$. Based on the clinical presentation, IVA can be classified into three distinct phenotypes: an acute neonatal form, a chronic intermittent form with late onset in childhood and a mild, potentially asymptomatic phenotype ${ }^{[10]}$. Patients with an acute neonatal presentation are at risk of severe, potentially life-threatening acidotic decompensations, showing a high mortality of $33 \%$. Patients with late onset is associated with developmental delay, who can also present acidotic episodes during catabolic factors such as infection and stress and appeared to have poor neurological outcomes ${ }^{[11,12]}$. In addition, even with early diagnosis and effective treatment, prognosis of IVA remains relatively disappointing. It has been reported that patients who have been metabolically stable for more than one decade were still at risk of metabolic decompensation in episodes of catabolic stress ${ }^{[13,14]}$.

Prenatal diagnosis is thus essential for the family with IVA proband to prevent the recurrence of IVA. Different approaches have been reported for the prenatal diagnosis of IVA, including genetic variant analysis of IVD genes in amniocytes ${ }^{[15]}$, IVD enzyme activity assay in amniotic or chorionic villi sampling ${ }^{[16]}$, and quantification of the characteristic metabolites such as acylcarnitines and organic acids in amniotic fluid (AF) ${ }^{[17,18]}$. However, each approach has its limitations. Mutation analysis depends entirely on complete genetic information from the proband and parents, while some probands were found to carry only one causative mutation, leading to the inability to make a precise diagnosis by genetic testing alone. Enzymatic analysis is available for prenatal diagnosis with cell cultivation required, which is troublesome and time-consuming. In addition, there is a risk of maternal cell contamination in above two methods which may potentially lead to misdiagnosis ${ }^{[19,20]}$. With the advancing use of mass spectrometry in metabolite analysis, this biochemical approach beneficially provides a fast and convenient method for the prenatal diagnosis of many inherited metabolic disorders ${ }^{[21-23]}$, yet limited research is available in IVA. In this study, we describe our experience in the prenatal diagnosis of IVA. Overall, we have investigated eight pregnancies in seven unrelated families by metabolite analysis together with genetic analysis in AF.

\section{Materials And Methods}




\section{Families and probands}

In this study, seven families (eight pregnancies) in which the probands diagnosed with IVA were referred to our center for prenatal diagnosis. The probands were diagnosed based on clinical symptoms, biochemical results and genetic testing of IVD genes.

Biochemical results included elevated levels of blood C5, C5/C2 and urinary IVG. The mutations spectrum of these probands are shown in Table 1. Informed consent forms were signed by the parents or legal guardians of the study participants. This study was approved by the Ethics Committee of Xinhua Hospital Affiliated to Shanghai Jiao Tong University School of Medicine (approval number XHEC-D-2021-172).

Table 1

The prenatal results of biochemical and genetic tests analysis in the amniotic fluid samples of 8 fetuses

\begin{tabular}{|c|c|c|c|c|c|c|c|c|c|}
\hline \multirow{3}{*}{$\begin{array}{l}\text { Fetal } \\
\text { sample } \\
\text { number }\end{array}$} & \multicolumn{3}{|c|}{ Metabolites of amniotic fluid } & \multicolumn{2}{|c|}{$\begin{array}{l}\text { Variants in the proband } \\
\text { (NM_002225) }\end{array}$} & \multirow[t]{3}{*}{$\begin{array}{l}\text { Fetus } \\
\text { status }\end{array}$} & \multicolumn{2}{|c|}{ Variant of fetus } & \\
\hline & \multicolumn{2}{|l|}{ MS/MS } & \multirow{2}{*}{$\begin{array}{l}\text { GS/MS } \\
\text { IVG } \\
(\mathrm{mmol} / \mathrm{mol} \\
\mathrm{cr})\end{array}$} & \multirow{2}{*}{$\begin{array}{l}\text { Allele } 1 \\
\text { (paternal) }\end{array}$} & \multirow{2}{*}{$\begin{array}{l}\text { Allele } 2 \\
\text { (maternal) }\end{array}$} & & & & \\
\hline & $\mathrm{C} 5(\mu \mathrm{mol} / \mathrm{L})$ & $\mathrm{C} 5 / \mathrm{C} 2$ & & & & & & & \\
\hline $\mathrm{F} 1 *$ & 0.28 & 0.02 & ND & & c. $149 \mathrm{G}>\mathrm{A}$ & c. $1184 \mathrm{G}>\mathrm{A}$ & Carrier & c. $1184 \mathrm{G}>\mathrm{A}$ & \\
\hline $\mathrm{F} 2 *$ & 0.24 & 0.02 & ND & & c. $149 \mathrm{G}>\mathrm{A}$ & c. $1184 \mathrm{G}>\mathrm{A}$ & Carrier & c. $1184 \mathrm{G}>\mathrm{A}$ & \\
\hline F3 & 0.31 & 0.03 & ND & & c. $205 \mathrm{G}>\mathrm{A}$ & $\begin{array}{l}\text { c.296- } \\
\text { 3_296- } \\
\text { 2delinsGG }\end{array}$ & Carrier & $\begin{array}{l}\text { c.296- } \\
\text { 3_296- } \\
\text { 2delinsGG }\end{array}$ & \\
\hline F4 & 0.946 & 0.08 & ND & & c. $415 \mathrm{C}>\mathrm{T}$ & c. $214 G>T$ & Carrier & c. $415 \mathrm{C}>\mathrm{T}$ & \\
\hline F5 & 0.66 & 0.06 & ND & & c. $359 \mathrm{G}>\mathrm{A}$ & c. $476 \mathrm{G}>\mathrm{C}$ & Carrier & c. $359 \mathrm{G}>\mathrm{A}$ & \\
\hline F6 & 2.7 & 0.072 & ND & & c. $476 \mathrm{G}>\mathrm{C}$ & $\mathrm{c} .358 \mathrm{C}>\mathrm{T}$ & Carrier & c. $358 \mathrm{C}>\mathrm{T}$ & \\
\hline F7 & 3.44 & 0.237 & ND & & Exon12del & c. $467 \mathrm{G}>\mathrm{C}$ & Affected & Exon12del & c. $467 \mathrm{G}>\mathrm{C}$ \\
\hline F8 & 4.22 & 0.48 & ND & & c. $548 \mathrm{C} \square \mathrm{T}$ & c.757A口G & Affected & c. $548 \mathrm{C} \square \mathrm{T}$ & c.757A $\square \mathrm{G}$ \\
\hline $\begin{array}{l}\text { Reference } \\
\text { range }\end{array}$ & $0.04-1.00$ & $\begin{array}{l}0.01- \\
0.08\end{array}$ & ND & & & & & & \\
\hline
\end{tabular}

\section{Amniocyte samples}

In each case, $20 \mathrm{ml}$ sample of AF was collected at 16-20 weeks of gestation from the pregnant women. The cells in $10 \mathrm{ml}$ AF was used for DNA extraction, and supernatant samples was used for metabolite analysis by mass spectrometry. The remaining $10 \mathrm{ml}$ of AF was cultured in a flask for karyotyping analysis, with the cultured amniocytes also available as a back-up. Furthermore, 3-4 ml of peripheral blood was collected from all IVA pedigree members to perform a linkage analysis and to exclude maternal cell contamination.

\section{Metabolite analysis}

The levels of C5 and C2 were quantitatively analyzed by MS/MS (Applied Biosystems, API 2000) using $3 \mu$ l of AF supernatant and the levels automatically calculated based on the assigned values for internal standards using Chemo View v1.2 software ${ }^{[24]}$. The organic acid fraction was extracted, methylated, and analyzed by chemical ionization GC/MS (QP2010, Shimadzu Limited, Kyoto, Japan) operated in selected ion monitoring mode. For each AF supernatant sample, $2 \mathrm{ml}$ of sample was mixed with stable isotope-labeled compounds and internal standards as described by Hasegawa et al ${ }^{[20]}$. Concentrations of IVG in the AF were calculated using GC-MS Solution v2.40 software.

In our study, the reference ranges of $\mathrm{C} 5, \mathrm{C} 5 / \mathrm{C} 2$ and IVG were $0.04-1.00 \mu \mathrm{mol} / \mathrm{L}, 0.01-0.08$ and $0.00 \mathrm{mmol} / \mathrm{mol} \mathrm{Cr}$, respectively. Fetuses with the above metabolites' levels higher than the upper limit of reference ranges were suggested as IVA. Standard scatter plots were 
generated for level of C5, C5/C2 and IVG in AFs by Prism 8 (Graph-Pad Software Inc.).

\section{Gene variant and linkage analysis}

Genomic DNA from the AF was extracted with a QIAamp DNA Blood Mini Kit (Qiagen Inc., Valencia, CA). Five closely linked flanking short tandem repeat (STR) markers at the IVD gene locus were selected to perform the linkage analysis and to exclude maternal cell contamination. We then conducted polymerase chain reaction (PCR) amplification and direct Sanger sequencing, which was performed as described in a previous paper ${ }^{[25]}$. Nucleotide variations were identified using a reference sequence from Genbank (IVD: NM_002225).

In our study, fetuses harbored homozygous variants or compound heterozygous variants of $I V D$ were diagnosed as IVA. We then used the ClinVar database, the HGMD database and the former literatures to identify whether the mutations had been reported.

\section{Results}

The genetic and biochemical results of the eight fetal samples from seven families are listed in Table 1. Among the eight fetuses, two fetuses were judged as "affected" and six fetuses were judged as "unaffected" based on the combination of these analyses. The STR analysis indicated that no samples had maternal cell contamination. Postnatal follow-up of all these six unaffected fetuses showed a normal phenotype. For the two affected fetuses, the parents chose to terminate pregnancies.

\section{Biochemical analysis of AF metabolites}

In our study, a total of two fetuses were found to have higher levels of C5 and C5/C2 compared to that of defined reference ranges and they were thus determined to be affected. Among the remaining six fetuses, the $\mathrm{C} 5$ level in F6 were slightly above the upper limit of the reference range, while the level of $\mathrm{C} 5 / \mathrm{C} 2$ were within the reference range. The other five samples showed normal levels of $\mathrm{C} 5$ and $\mathrm{C} 5 / \mathrm{C} 2$. And these six fetuses were determined to be unaffected according to our metabolite test results. However, the IVG levels in all eight fetal samples could not be detected at all (Figure 1).

\section{Genetic analysis of the pathogenic variants in amniocyte DNA}

Luckily, all the eight at-risk fetuses had clear information concerning pathogenic variants in the probands and parents. The molecular spectrum of this cohort was heterogeneous, with thirteen different variants identified: c.149G>A, c.205G>A, c.214G>T, c.358C>T, c.359G >A, c.415C>T, c.467G >C, c.476G>C, c.548C口T, c.757A口G, c.1184G>A, c.296-3_296-2delinsGG and Exon12del, most of which were missense variant and the c.214G>T, c.358C>T, c.415C>T and c.296-3_296-2delinsGG variants were novel.

In this study, a total of two fetuses harboring compound heterozygous variants, were determined to be affected. And the remaining six fetuses with a pathogenic variant were determined to be unaffected as heterozygous carriers.

\section{Comparison of metabolite results and genetic results}

Since the level of IVG could not be detected in all samples, we mainly compared metabolite results of C5 and C5/C2 with genetic results, which showed high consistency in our study. A total of two fetuses were determined to be affected according their genetic results, both of whom carried compound heterozygous variants. These two fetuses were found to have elevated levels of C5 and $\mathrm{C} 5 / \mathrm{C} 2$. Thus, they were also judged as affected by metabolite results (Table 1). The remaining six fetuses were determined to be unaffected by mutation analysis, all of whom were IVD pathogenic single variant carriers. Among them, five fetuses were found to have normal levels of $\mathrm{C} 5$ and $\mathrm{C} 5 / \mathrm{C} 2$ while the other one fetus (F6) showed slightly inconsistent genetic and metabolite results (Table 1). The fetus with a pathogenic variant inherited from the mother was found to have an elevated level of $C 5$, but a normal level of $\mathrm{C} 5 / \mathrm{C} 2$. This fetus was determined to be unaffected based on the combination of genetic and metabolite results. Postnatal follow-up of the fetus showed a normal phenotype.

The comparison of metabolite results with genetic results revealed that the sensitivities of $\mathrm{C} 5$ and $\mathrm{C} 5 / \mathrm{C} 2$ were both $100 \%$, and the specificities were $83.3 \%$ and $100 \%$, respectively. The positive predicative values of C5 and C5/C2 were $66.7 \%$ and $100 \%$, respectively, and the negative predicative values were $83.3 \%$ and $100 \%$, respectively.

\section{Discussion}


IVA is known as a relatively rare, potentially life-threatening inborn error of leucine metabolism. Even with rapid diagnosis and effective treatment, patients with IVA are still at risk of metabolic decompensation, no matter what the form is. The disappointing outcome will increase the families economic and spiritual burden. Thus, prenatal diagnosis is an essential strategy for the family with IVA probands to prevent the recurrence of IVA. In this study, we shared our experiences with prenatal diagnosis of IVA in eight pregnancies.

The measurement of metabolite levels in AF by mass spectrometry has been increasingly used for the prenatal diagnosis of numerous inherited metabolic disorders, yet few reported in IVA. In a notable example, Shigematsu et al. reported that metabolite analysis of acylcarnitines by MS/MS and organic acids by GC/MS in AF allowed a rapid and reliable diagnosis, while it was based on a single case $^{[17]}$. In addition, it has been reported that IVG possibly present below the lowest levels, which remains a risk of false-negative results ${ }^{[26]}$. Therefore, systematic application and evaluation of these biochemical methods are critical. Given that genetic analysis was generally recognized as the golden standard for prenatal diagnosis of IVA, in this study, we retrospectively reviewed prenatal diagnostic data from eight at-risk pregnancies and first analyzed the reliability of the biochemical approach for prenatal diagnosis of IVA by comparing the biochemical results with the molecular results.

Among these eight cases, the levels of $\mathrm{C} 5$ and $\mathrm{C} 5 / \mathrm{C} 2$ were consistent with genetic results in both affected fetuses and in five of six unaffected fetuses, these two biochemical markers were also completely consistent with genetic results. Only the level of C5 in F6 was found to show a slight discrepancy with genetic results. One of the probable reasons might be associated with the selection of the cutoff value. It has been suggested that a reference range based exclusively on normal population might lead to many false positive results ${ }^{[27]}$. Therefore, the reference range of prenatal metabolite levels need to be adjusted in response to the overlap between normal population and disorder range. However, it is difficult to establish more reasonable ranges on the basis of the small sample size in our study. The IVG assay was also performed for the prenatal diagnosis of IVA, which yet was found not to be valuable, since the AFs from all eight at-risk pregnancies contained no measurable IVG, whether they carried an affected or unaffected fetus. This is inconsistent with that described in the previous reports where the IVG levels in affected fetuses were notably 30-60 times higher than that in unaffected fetuses ${ }^{[16,18]}$. The false negative results might be due to the low presentation of this metabolite in AFs and adsorption losses. According to our data and comparisons of the metabolite results and genetic results, the sensitivities of $\mathrm{C} 5$ and C5/C2 were both $100 \%$, and the specificities of C5 and C5/C2 were $83.3 \%$ and $100 \%$, respectively. Therefore, C5/C2 appeared to be the most reliable of these three tested biochemical markers. Due to the limitation of sample size in this study, further data are needed to determine the reliability of these three biochemical markers in the prenatal diagnosis of IVA.

Mutation analysis is generally recognized as the most reliable strategy for prenatal diagnosis of IVA, which yet may potentially lead to false negative results due to maternal cell contamination. Another limitation of this method is that it depends on the availability of known familial mutations. Luckily, all the probands in our center had found two causative variants and no samples had maternal cell contamination. However, in some IVA families, not more than one causative mutation was found in the proband, or genetic testing was not performed, especially in some remote areas, this can hinder a precise diagnosis by genetic analysis alone. In this situation, metabolite analysis by mass spectrometry could be optional which is able to provide reliable results using only a small amount of sample. Additionally, the biochemical results can be achieved within three days, which is much earlier than that of genetic analysis taking at least two weeks. This enables IVA families to make a timely decision concerning the pregnancies. This advantage was observed in our previous reports on prenatal metabolite analysis in methylmalonic acidemia, propionic acidemia and glutaric acidemia- $\rrbracket^{[21-23]}$. Therefore, despite the accuracy of genetic testing, biochemical analysis appeared to be a reliable method in the prenatal diagnosis of IVA families, especially when genetic results were inconclusive, and this method could also allow a more precise diagnosis in conjunction with mutation analysis. However, the reliability of biochemical approach in the prenatal diagnosis of IVA is warranted to be further verified by expanding the sample size.

\section{Conclusion}

In summary, prenatal diagnosis is necessary in families of probands with IVA. Biochemical analysis using only a small amount of amniotic fluid, offers fast and reliable results, which could be a suitable option in the prenatal diagnosis of IVA, especially in families without conclusive genetic results, and can increase the accuracy together with mutation analysis. Among the three tested biochemical markers, C5/C2 appeared to the most reliable, which yield a sensitivity of $100 \%$ and specificity of $100 \%$.

\section{Abbreviations}


IVA

Isovaleric acidemia

IVD

Isovaleryl-CoA dehydrogenase

\section{Declarations}

\section{Acknowledgements}

We acknowledge all patients and their family for participating in this study.

\section{Authors' contributions}

All authors read and approved the final manuscript. LSH and LW: study concept and design. SD and LLL: acquisition and analysis of data; drafting of the manuscript. FX, WJJ: performed metabolite test and analysis; ZWG: performed genetic testing of AFs; LSH, LW, WJQ, HWZ, BX, LLL, LPD and XFG: study supervision; in charge of the patients after referral to each center and further diagnosis.

\section{Funding}

Scientific research Project plan of Shanghai Municipal Health Commission (No.202040448, No.202140346).

\section{Availability of data and materials}

All data generated or analyzed during this study are included in this published article and its supplementary information files.

\section{Ethics approval and consent to participate}

Written informed consent was obtained from all participants, and our study was approved by the Ethics Committee of Xinhua Hospital (Approval No. XHEC-D-2021-172).

\section{Consent for publication}

Participants provided written informed consent.

\section{Conflicts of interest}

None declared.

\section{Authors' information}

${ }^{1}$ Department of Pediatric Endocrinology and Genetic Metabolism, Xinhua Hospital, Shanghai Institute of Pediatric Research, Shanghai Jiao Tong University School of Medicine, Shanghai, China

${ }^{2}$ Center for Prenatal Diagnosis, Xinhua Hospital, Shanghai Jiao Tong University School of Medicine, Shanghai, China.

${ }^{3}$ Neonatal Disease Screening Center, Zibo Maternal and Child Health Hospital, Zibo, China

\section{References}

1. Golbahar J, Al-Jishi EA, Altayab DD, et al. Selective newborn screening of inborn errors of amino acids, organic acids and fatty acids metabolism in the Kingdom of Bahrain [J]. Mol Genet Metab. 2013;110(1-2):98-101.

2. Kasper DC, Ratschmann R, Metz TF, et al. The national Austrian newborn screening program - eight years experience with mass spectrometry. past, present, and future goals [J]. Wien Klin Wochenschr. 2010;122(21-22):607-13.

3. Chace DH, Kalas TA, Naylor EW. Use of tandem mass spectrometry for multianalyte screening of dried blood specimens from newborns [J]. Clin Chem. 2003;49(11):1797-817. 
4. Lin WD, Wang CH, Lee CC, et al. Genetic mutation profile of isovaleric acidemia patients in Taiwan [J]. Mol Genet Metab. 2007;90(2):134-9.

5. Hu ZZ, Yang JB, Hu LW, et al. Screening and clinical analysis of isovaleric acidemia newborn in Zhejiang province. Journal of Zhejiang University(Medical Sciences). 2020;49(5):556-64.

6. Lin Y, Chen D, Peng W, et al. Newborn screening for isovaleric acidemia in Quanzhou, China [J]. Clin Chim Acta. 2020;509:25-9.

7. Tanaka K, Budd MA, Efron ML, et al. Isovaleric acidemia: a new genetic defect of leucine metabolism [J]. Proc Natl Acad Sci U S A. 1966;56(1):236-42.

8. Ibarra-González I, Fernández-Lainez C, Guillén-López S, et al. Molecular analysis using targeted next generation DNA sequencing and clinical spectrum of Mexican patients with isovaleric acidemia [J]. Clin Chim Acta. 2020;501:216-21.

9. Vockley J, Ensenauer R. Isovaleric acidemia: new aspects of genetic and phenotypic heterogeneity [J]. Am J Med Genet C Semin Med Genet. 2006;142c(2):95-103.

10. Ensenauer R, Vockley J, Willard JM, et al. A common mutation is associated with a mild, potentially asymptomatic phenotype in patients with isovaleric acidemia diagnosed by newborn screening [J]. Am J Hum Genet. 2004;75(6):1136-42.

11. Grünert SC, Wendel U, Lindner M, et al. Clinical and neurocognitive outcome in symptomatic isovaleric acidemia [J]. Orphanet J Rare Dis. 2012;7:9.

12. Kölker S, Valayannopoulos V, Burlina AB, et al. The phenotypic spectrum of organic acidurias and urea cycle disorders. Part 2: the evolving clinical phenotype [J]. J Inherit Metab Dis. 2015;38(6):1059-74.

13. Kimmoun A, Abboud G, Strazeck J, et al. Acute decompensation of isovaleric acidemia induced by Graves' disease [J]. Intensive care medicine. 2008;34(12):2315-6.

14. Szymańska E, Jezela-Stanek A, Bogdańska A, et al. Long Term Follow-Up of Polish Patients with Isovaleric Aciduria. Clinical and Molecular Delineation of Isovaleric Aciduria [J]. Diagnostics (Basel). 2020;10:10.

15. Tanacan A, Gurbuz BB, Aydin E, et al. Prenatal Diagnosis of Organic Acidemias at a Tertiary Center [J]. Balkan J Med Genet. 2019;22(1):29-34.

16. Kleijer WJ, van der Kraan M, Huijmans JG, et al. Prenatal diagnosis of isovaleric acidaemia by enzyme and metabolite assay in the first and second trimesters [J]. Prenat Diagn. 1995;15(6):527-33.

17. Shigematsu Y, Kikawa Y, Sudo M, et al. Prenatal diagnosis of isovaleric acidemia by fast atom bombardment and tandem mass spectrometry [J]. Clin Chim Acta. 1991;203(2-3):369-74.

18. Hine DG, Hack AM, Goodman SI, et al. Stable isotope dilution analysis of isovalerylglycine in amniotic fluid and urine and its application for the prenatal diagnosis of isovaleric acidemia [J]. Pediatr Res. 1986;20(3):222-6.

19. Roberts E, Duckett DP, Lang GD. Maternal cell contamination in chorionic villus samples assessed by direct preparations and three different culture methods [J]. Prenat Diagn. 1988;8(9):635-40.

20. Hasegawa $Y$, Iga M, Kimura $M$, et al. Prenatal diagnosis for organic acid disorders using two mass spectrometric methods, gas chromatography mass spectrometry and tandem mass spectrometry [J]. J Chromatogr B Analyt Technol Biomed Life Sci. 2005;823(1):13-7.

21. Dai $\mathrm{M}$, Xiao $B$, Zhang $\mathrm{H}$, et al. Biochemical and genetic approaches to the prenatal diagnosis of propionic acidemia in 78 pregnancies [J]. Orphanet J Rare Dis. 2020;15(1):276.

22. Ji X, Wang H, Ye J, et al. Prenatal diagnosis of methylmalonic aciduria from amniotic fluid using genetic and biochemical approaches [J]. Prenat Diagn. 2019;39(11):993-7.

23. Xiao B, Qiu W, Ye J, et al. Prenatal Diagnosis of Glutaric Acidemia I Based on Amniotic Fluid Samples in 42 Families Using Genetic and Biochemical Approaches [J]. Front Genet. 2020;11:496.

24. Han LS, Ye J, Qiu WJ, et al. Selective screening for inborn errors of metabolism on clinical patients using tandem mass spectrometry in China: a four-year report [J]. J Inherit Metab Dis. 2007;30(4):507-14.

25. Lee YW, Lee DH, Vockley J, et al. Different spectrum of mutations of isovaleryl-CoA dehydrogenase (IVD) gene in Korean patients with isovaleric acidemia [J]. Mol Genet Metab. 2007;92(1-2):71-7.

26. Kumps A, Vamos E, Mardens $Y$, et al. Assessment of an electron-impact GC-MS method for organic acids and glycine conjugates in amniotic fluid [J]. J Inherit Metab Dis. 2004;27(5):567-79. 
27. McHugh D, Cameron CA, Abdenur JE, et al. Clinical validation of cutoff target ranges in newborn screening of metabolic disorders by tandem mass spectrometry: a worldwide collaborative project [J]. Genet Med. 2011;13(3):230-54.

\section{Figures}

A

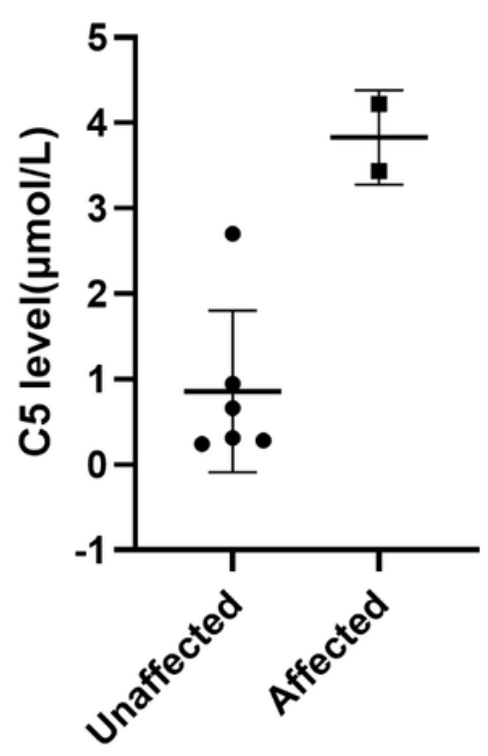

B

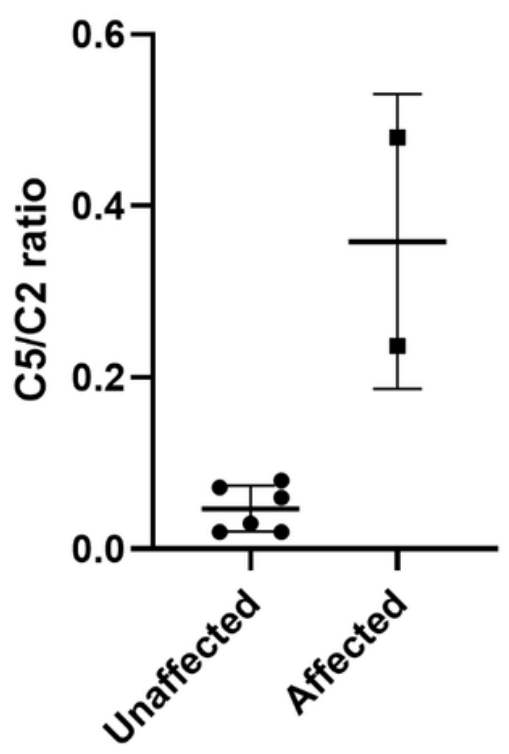

C

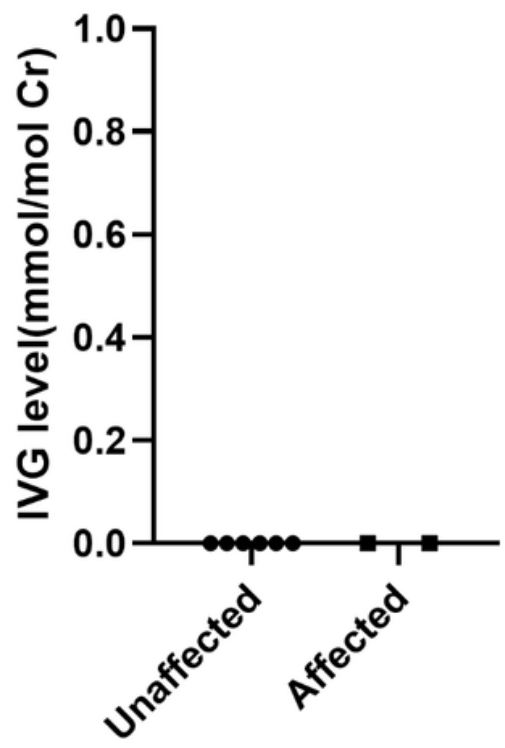

Figure 1

Scatter-plots of individual levels of C5 (A), C5/C2 (B) and IVG (C) in the supernatant of AFs of 6 unaffected and 2 affected fetuses. 